\title{
ADHESIVE BONDING VIA EXPOSURE TO VARIABLE FREQUENCY MICROWAVE RADIATION
}

\author{
Felix L. Paulauskas, April D. McMillan, C. David Warren \\ Oak Ridge National Laboratory
}

\author{
Spring Materials Research Society (MRS) - Meeting \\ San Francisco, California, April 8-12, 1996
}

\begin{abstract}
"The submitted manuscript has been authored by a contractor of the U.S. Government under contract No. DE-AC05960R22464. Accordingly, the U.S. Government retains a nonexclusive, royalty-free license to publish or reproduce the published form of this contribution, or allow others to do so, for U.S. Government purposes."
\end{abstract}

Research was performed at Oak Ridge National Laboratory and sponsored by the Office of Transportation materials, U.S. Department of Energy under contract DE-AC05-960R22464 with Lockheed Martin Energy Research Corp.

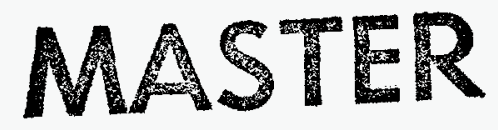




\section{DISCLAIMER}

Portions of this document may be illegible in electronic image products. Images are produced from the best available original document. 


\title{
ADHESIVE BONDING VIA EXPOSURE TO VARIABLE FREQUENCY MICROWAVE RADIATION
}

\author{
FELIX L. PAULAUSKAS, APRIL D. McMILLAN and C. DAVID WARREN \\ Oak Ridge National Laboratory, P.O. Box 2009, Oak Ridge, Tennessee 37831-8048
}

\begin{abstract}
Adhesive bonding through the application of variable frequency microwave (VFM) radiation has been evaluated as an alternative curing method for joining composite materials. The studies showed that the required cure time of a thermosetting epoxy adhesive is substantially reduced by the use of VFM when compared to conventional (thermal) curing methods. Variable frequency microwave processing appeared to yield a slight reduction in the required adhesive cure time when compared to processing by the application of single frequency microwave radiation. In contrast to the single frequency processing, the variable frequency methodology does not readily produce localized overheating (burnt or brown spots) in the adhesive or the composite. This makes handling and location of the sample in the microwave oven less critical for producing high quality bonds and allows for a more homogeneous distribution of the cure energy. Variable frequency microwave processing is a valuable alternative method for rapidly curing thermoset adhesives at low input power levels.
\end{abstract}

KEY WORDS: Variable Frequency Microwave, Composite Bonding, Adhesive.

\section{INTRODUCTION}

In the past few years, Oak Ridge National Laboratory (ORNL) has been involved in a national initiative to conduct research aimed at promoting the use of lighter weight materials in automotive structures for the purpose of increasing fuel efficiency and reducing environmental pollutant emissions [1]. Key to this initiative is the joining of non-ferrous materials. The most promising method for joining the wide spectrum of alternate materials that may see automotive implementation is adhesive bonding. Unfortunately, the implementation of adhesives on the manufacturing plant floor suffers from a significant economical obstacle. Structural adhesives often require long cure times which translate into elevated per part costs. In order to make the use of adhesives for primary structures a reality in high production rate consumer goods industries, technologies for reducing the cure time of the adhesive must be discovered, developed and deployed. Variable frequency microwave radiation may be one solution to this problem.

This investigation pertains to the effect of variable frequency microwave radiation on the cure time and mechanical properties of an epoxy adhesive. Mechanical properties were evaluated by single lap-shear testing. The VFM samples were compared with data obtained via conventional (thermal) processing and single (fixed) frequency microwave curing of similar samples. It was found that cure time is substantially reduced by VFM radiation, when compared to conventional curing techniques. It was also found that VFM radiation curing 
yielded only a slight reduction in cure time when compared to single frequency microwave radiation processing but did so without producing localized overheating.

The substrate materials evaluated in this work are glass and a urethane-based composite with glass fiber reinforcement (SRIM-part). Glass substrates (annealed soda lime glass microscope slides) were bonded via microwave radiation curing of an epoxy adhesive to eliminate the variable represented by the absorption of microwave energy by substrates. The SRIM composite is a glass reinforced isocyanurate, DOW MM364, which is currently being investigated for use by the domestic automotive industry. The glass mat is a random swirled glass with a U750 binder and comprises $55 \mathrm{wt} \%$ of the composite. The adhesive tested in this work is the BF Goodrich 582E which is a toughened epoxy currently being investigated for structural automotive applications.

The power distribution within a microwave cavity resulting from a fixed frequency source is extremely heterogeneous. As is shown in Figure 1A, a single frequency source yields regions of very high energy and other regions of very low energy within any given cavity plane. If a different plane is chosen within the microwave cavity, a totally different energy profile will exist. By changing any one of many variables including the frequency, cavity size, input power, etc., totally different energy profiles may be achieved but each would have the same characteristic non-uniformity. Adhesives being cured by microwave energy that are subjected to these heterogeneous power distributions would have regions that are well cured, areas that are barely cross-linked and other sectors which are over cured (burnt). One method to overcome this problem when using fixed frequency sources is by placing the specimens on a rotating plate during irradiation or to have deflectors in the cavity rotating during the curing process.

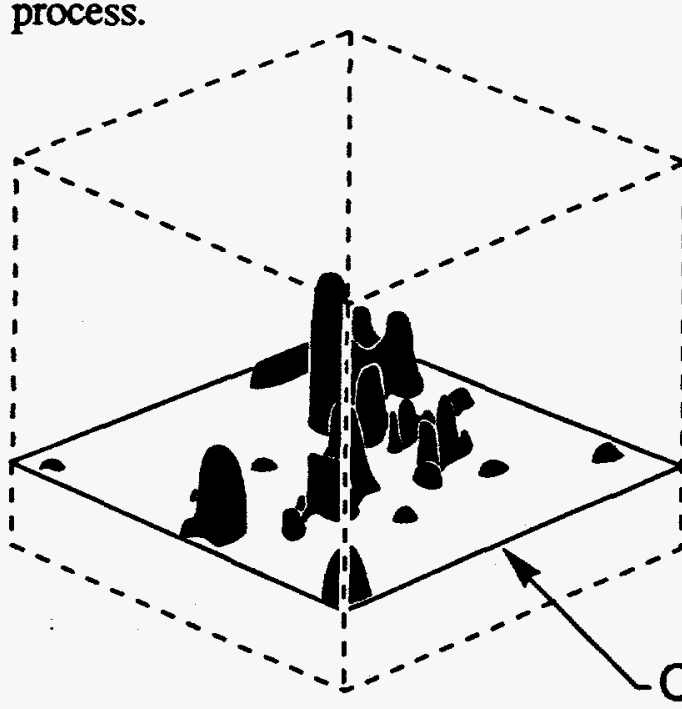

(A)
Plane In Observation

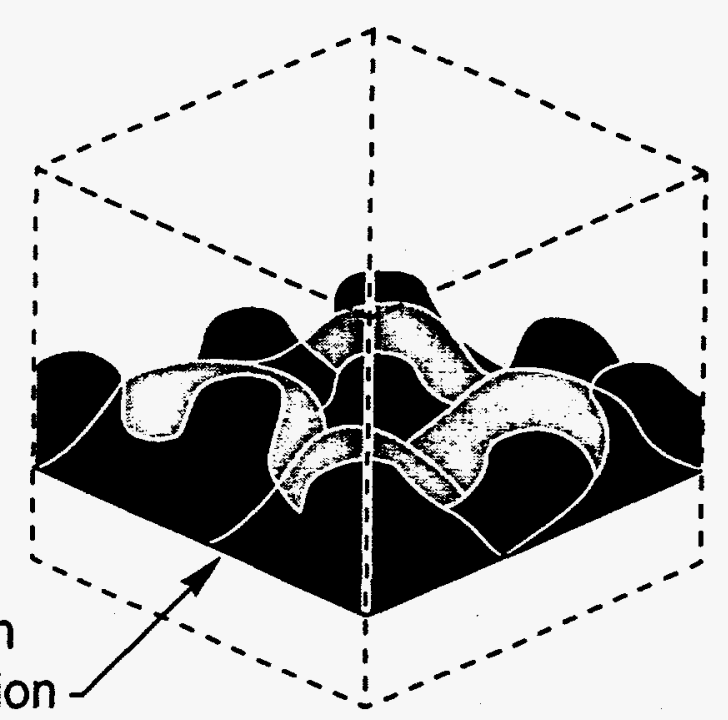

(B)

Figure 1. (A) Typical energy distribution in a single frequency microwave oven. (B) Typical energy distribution in a variable frequency microwave cavity. Note that the cumulative effect of many energy profiles in the VFM results in a homogenization of the total energy distribution.

Variable frequency microwave techniques are based on the concept of identifying a central frequency and rapidly sweeping the microwave energy over a range of frequencies greater than and less than the central frequency creating a range of effective frequencies. Since each 
instantaneous frequency has a different electric field and subsequently a different energy deposition profile, the sweeping of frequencies overlays the energy distribution profiles of many frequencies. The cumulative effect of all these instantaneous but different modes will result in a homogenization of the total energy deposition in the material being processed as is schematically indicated in Figure 1B. Theoretical models and experimental results $[2,3,4,5]$ corroborate this effect. The uniform energy distribution allows for even curing within materials in the cavity. The result is that adhesive samples can be cured in a more homogenous manner, without burnt spots and without uncured regions. Generally, a variable frequency microwave can be made to function as a single frequency source by choosing a center frequency and making the frequency range equal to zero. By doing this the center frequency becomes the fixed frequency.

\section{EXPERIMENTAL PROCEDURE}

To determine the correlation between cure times and input power for single frequency microwave irradiation, glass slides were joined using the epoxy adhesive and four to five 30 mil ( 0.030 inch) glass beads strategically dispersed in each uncured joint to accurately control the bondline thickness. Subsequently, all samples were exposed to varying power levels of microwave radiation using a Cober SF6 power supply which provided up to $5.5 \mathrm{kw}$ of fixed $2.45 \mathrm{GHz}$ radiation in a $61 \times 61 \times 61 \mathrm{~cm}$ multi-mode cavity. Samples were visually inspected to determine the degree of polymerization (crosslinking) by noting the color changes and also hardness of the adhesive. This adhesive changes from olive to bright green when crosslinking is well advanced. Results were verified by differential scanning calorimeter (DSC) testing of two or three samples for proper cure. Curves were then constructed for each power level relating input power to required curing time. This process was then repeated for the isocyanurate composite using the same processing conditions.

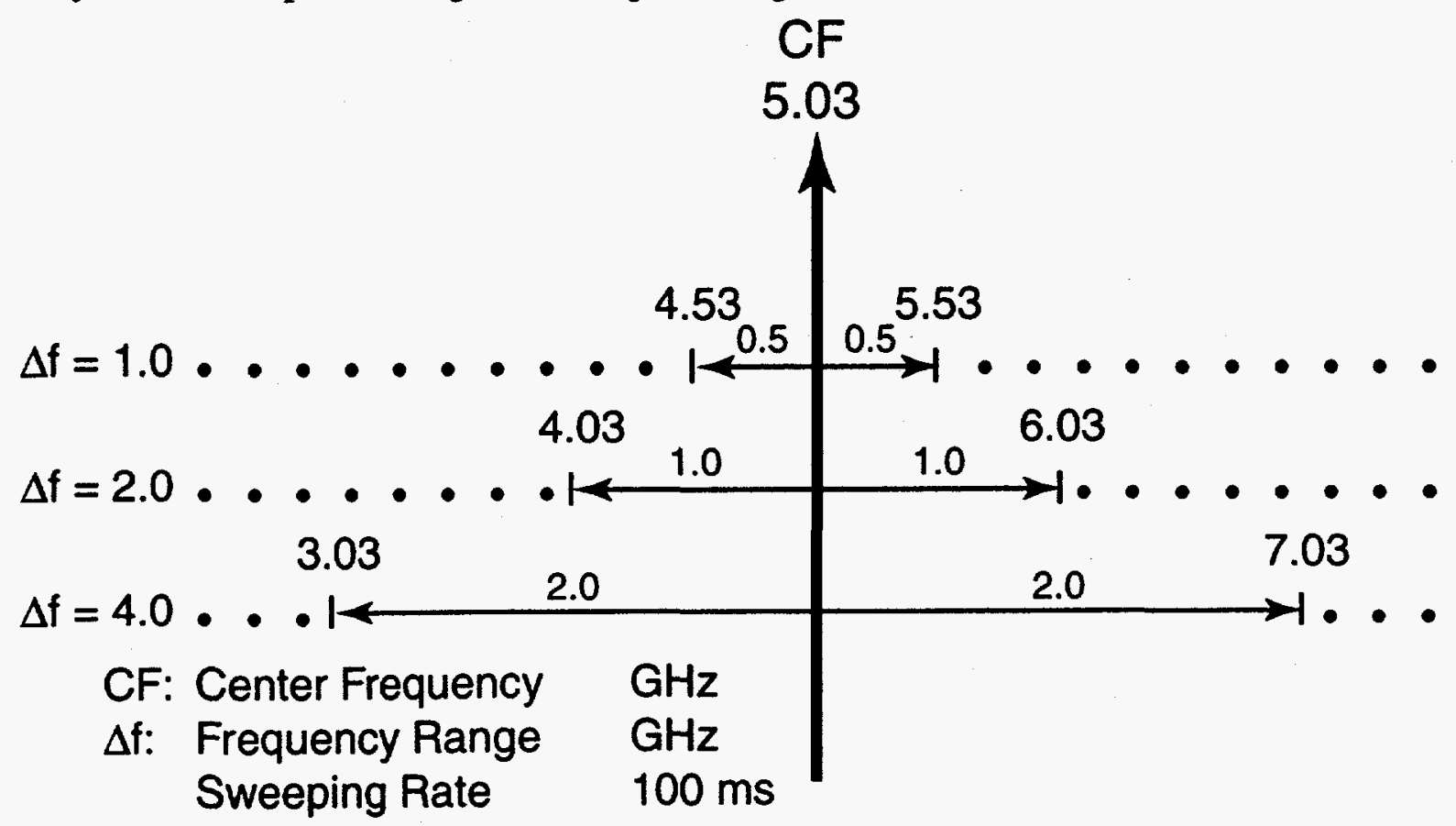

Figure 2. Variable Frequency Microwave Processing Spectrum 
To determine the relationship between the required cure time and the input power for variable frequency microwave processing, a center frequency for the VFM of $5.03 \mathrm{GHz}$ was selected. Frequency ranges of $1.0,2.0$, and $4.0 \mathrm{GHz}$ were chosen and a sweep rate of $100 \mathrm{~ms}$ was employed as is shown in Figure 2. For each frequency range, identical glass slide adherents were joined using the same adhesive. Four to five 30 mil $(0.030$ inch $)$ glass beads selectively placed in the joint to accurately control the bondline thickness. By a trial and error approach, the cure time required for each frequency sweep range was identified. Once this was accomplished for the glass slides with the absence of lossy effects, it was repeated for the composite material under the same conditions (center frequency, frequency ranges, sweep rate, adhesive, bondline thickness and sample preparation procedures).

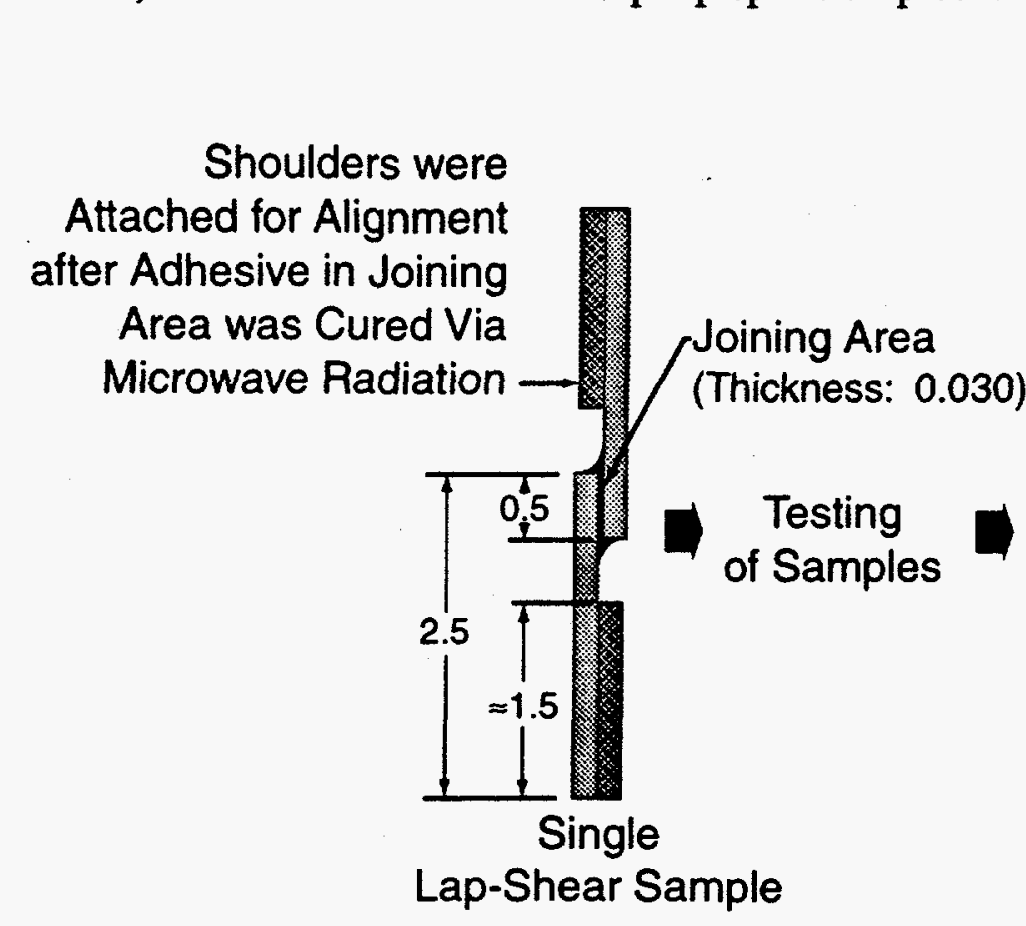

All measurements in inches.

(A)

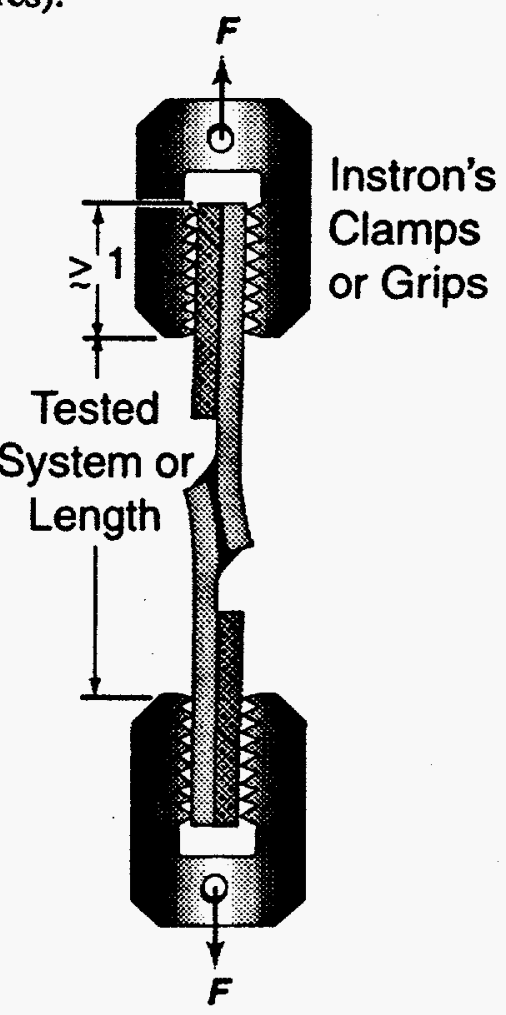

(B)

Figure 3. (A) As-Processed Specimen and Joint Configuration for the Single Lap Shear Tests. (B) System Deformation and Displacement in the Single Lap Shear Test

The characterization of the bond strength in the processed samples was determined using single lap shear samples. Composite specimens 2.5 inches long and 1.0 inch wide were bonded to form single lap shear joints with a 0.5 inch overlap as is shown in Figure 3. Glass beads were also used in the lap shear samples to hold a consistent bondline thickness. For single frequency microwave processing four distinct power level and curing time combinations were selected by preliminary trials and multiple specimens fabricated. The maximum power selected was 1200 Watts which corresponds to the shortest required curing time. Higher input powers caused the adhesive to be expelled from the joint after only a short irradiation. This may be related to a rapid exotherm or localized overheating in the adhesive due to a too fast deposition of energy into the epoxy prior to completion of the crosslinking reaction. In an attempt to further reduce the curing time while avoiding the exotherm, a two step, two energy level curing 
method was employed. The curing process was started with a high level of input power for a very short processing time and subsequently the power was reduced to a lower level and an appropriate processing time was given to complete the crosslinking process. This process was repeated for several high and low power level combinations and is presented in other work [6].

For the variable frequency microwave processing trials, a $5.03 \mathrm{GHz}$ center frequency was selected with frequency sweeps of 1.0, 2.0 and $4.0 \mathrm{GHz}$ employed. Because of the intrinsic nature of the VFM oven, the maximum deliverable forward power is decreased with an increase in the size of the frequency range. Average forward power was about $190 \mathrm{~W}$ for $A^{f}=1 \mathrm{GHz}$, $160 \mathrm{~W}$ for $\Delta \mathrm{f}=2 \mathrm{GHz}$ and about $130 \mathrm{~W}$ for $\Delta \mathrm{f}=4 \mathrm{GHz}$. The VFM equipment utilized in this project was Lambda Technologies Inc., Model T-4001E, a multimode cavity which is capable of delivering forward power at ranges over 2.5 to $7.5 \mathrm{GHz}$ or 7.5 to $17.5 \mathrm{GHz}$ and has a nominal maximum forward power of 200 watts. Equal numbers of samples were processed for each processing condition for both single and variable frequency microwave studies.

A control group of conventionally (thermally) cured samples was fabricated where the samples were cured for 45 minutes at $150^{\circ} \mathrm{C}$. For all specimens (conventional and microwave processed), the urethane substrate was subjected to a drying cycle of $101^{\circ} \mathrm{C}$ for 48 hours prior to bonding. This was done to eliminate the interaction of residual water on the substrate with the incident microwave energy. The drying operation [7] is also a secondary post-cure operation for this material which completes the cure of any non-crosslinked material. This post cure could likely be accomplished directly with the microwave if processing conditions are properly designed.

To aid in better alignment and to minimize bending, 1.5 inch shoulders were bonded on each specimen as is depicted in Figure 3. Lap shear tests were then conducted on all samples using a Model 1125 Instron tensile testing machine and a crosshead displacement rate of 0.05 inches per minute. The complex test geometry of the single lap shear specimen imparts stresses to the substrates and adhesives which are difficult to define. Failure normally occurs due to peel (Mode I) stresses rather than shear (Mode II) stresses as the test name implies. Similarly, strain is also difficult to relate to machine displacement for the single lap shear test due to the complex test geometry, specimen geometry and off-set load path [8].

\section{SINGLE FREQUENCY MICROWAVE RESULTS}

\section{Microwave Processing}

The first system evaluated was glass slides adhesively bonded using single frequency microwave $(2.45 \mathrm{GHz}$ ) radiation over a range of input powers. An analysis of the data reveals that the epoxy cure time approximately followed the power law theory in that as electric field intensity increased, cure time decreased [6]. The empirical relationship indicated by the experimental data is that the power (P) varies as the inverse power of the cure time of the adhesive for this material combination. In the high power region, the experimental data indicates that the power is the inverse square root of the cure time, while for the lower input power region, the input power is inversely proportional to the exposure or cure time. Through analyzing the experimental data presented in Figure 4, an appropriate empirical function was determined which describes this data to a high degree of accuracy. The empirical relationship which describes the observed data is a power law function with an exponent of -0.612 and a constant term of 1464 . 


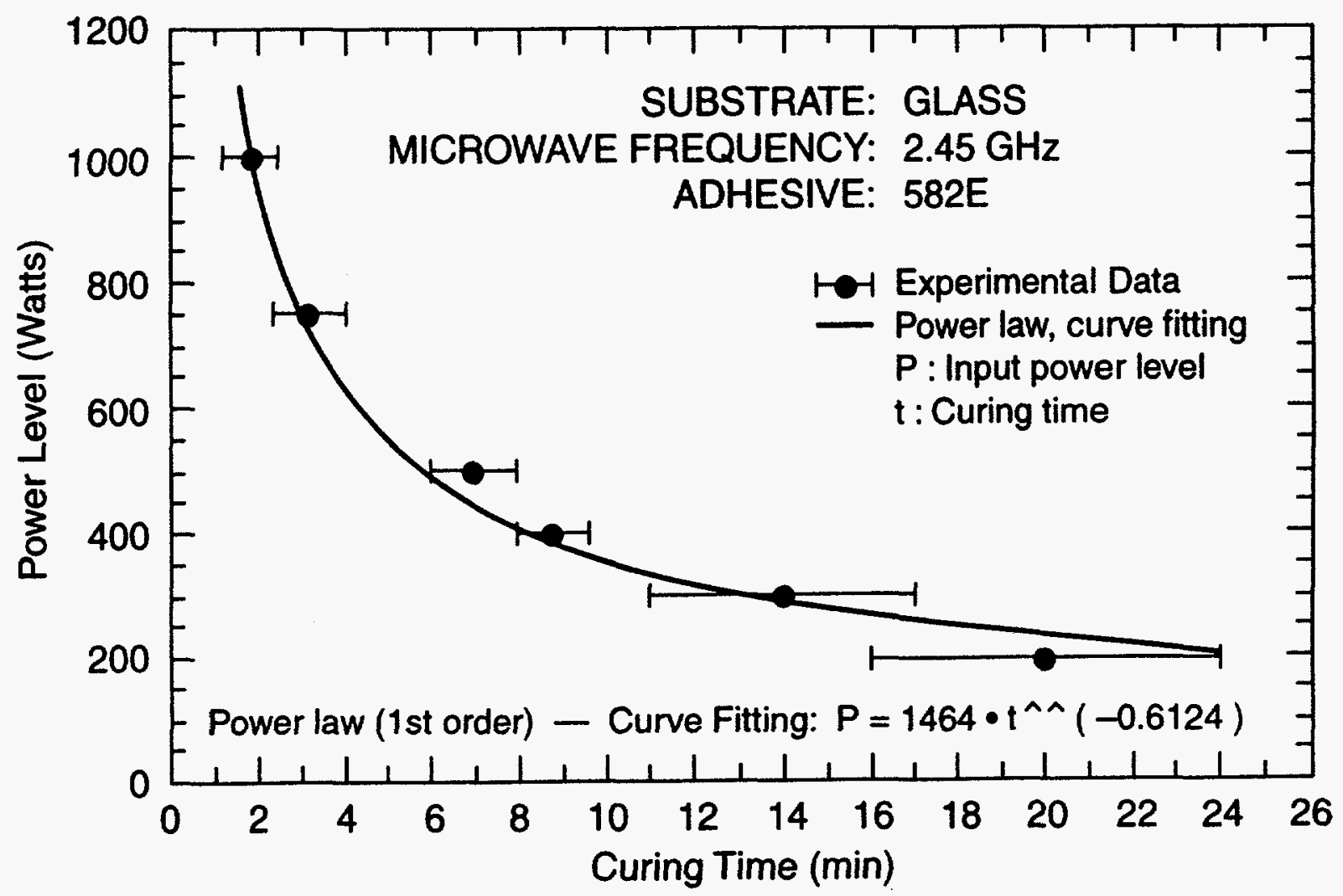

Figure 4. Cure Time vs. Power Level for the Adhesive on the Glass Substrates. Power Law Approximation.

Cure times below six minutes, which correspond to input powers about 500 watts, produced samples with a significant volume fraction of bubbles in the as-cured adhesive. It is likely that the energy deposition into these samples was too rapid resulting in excessive heating of the adhesive prior to sufficient crosslinking to prevent the flow of the adhesive. As a result, there exists an upper threshold energy for curing this adhesive. As the input energy was decreased, the time required for complete crosslinking was increased. At sufficiently low power inputs crosslinking would not be promoted. For this material system the lower threshold is below 150 watts. Therefore for this material and microwave system the effective curing range of the adhesives is between 150 and 500 watts and the complete cure can be achieved in as little as six minutes.

The second system evaluated was the glass fiber reinforced isocyanurate composite previously described. The composite was adhesively bonded using single frequency microwave $(2.45 \mathrm{GHz})$ radiation over a range of input powers. An analysis of the data show that this material system also approximately follows the power law theory which is shown in Figure 5. The empirical function which best describes this data is a power law function with an exponent of -0.503 and a constant term of 1410 .

Input power of about 550 watts, which corresponds to a cure time of about eight minutes, is the maximum input power limit due to the formation of bubbles in the adhesive. The lower limit for complete crosslinking for this material system is below approximately $\mathbf{3 0 0}$ watts. Therefore for this material and microwave system the effective curing range of the adhesive is between 300 and 550 watts and the complete cure can be achieved in as little as eight minutes. It is not surprising that the urethane substrates had higher input power limits and required 
substrates bonded with the same adhesive and irradiated at the same frequency. The difference between the lossy characteristics of the two substrates would require more energy and longer cure times due to absorption of a portion of the input energy by the composite.

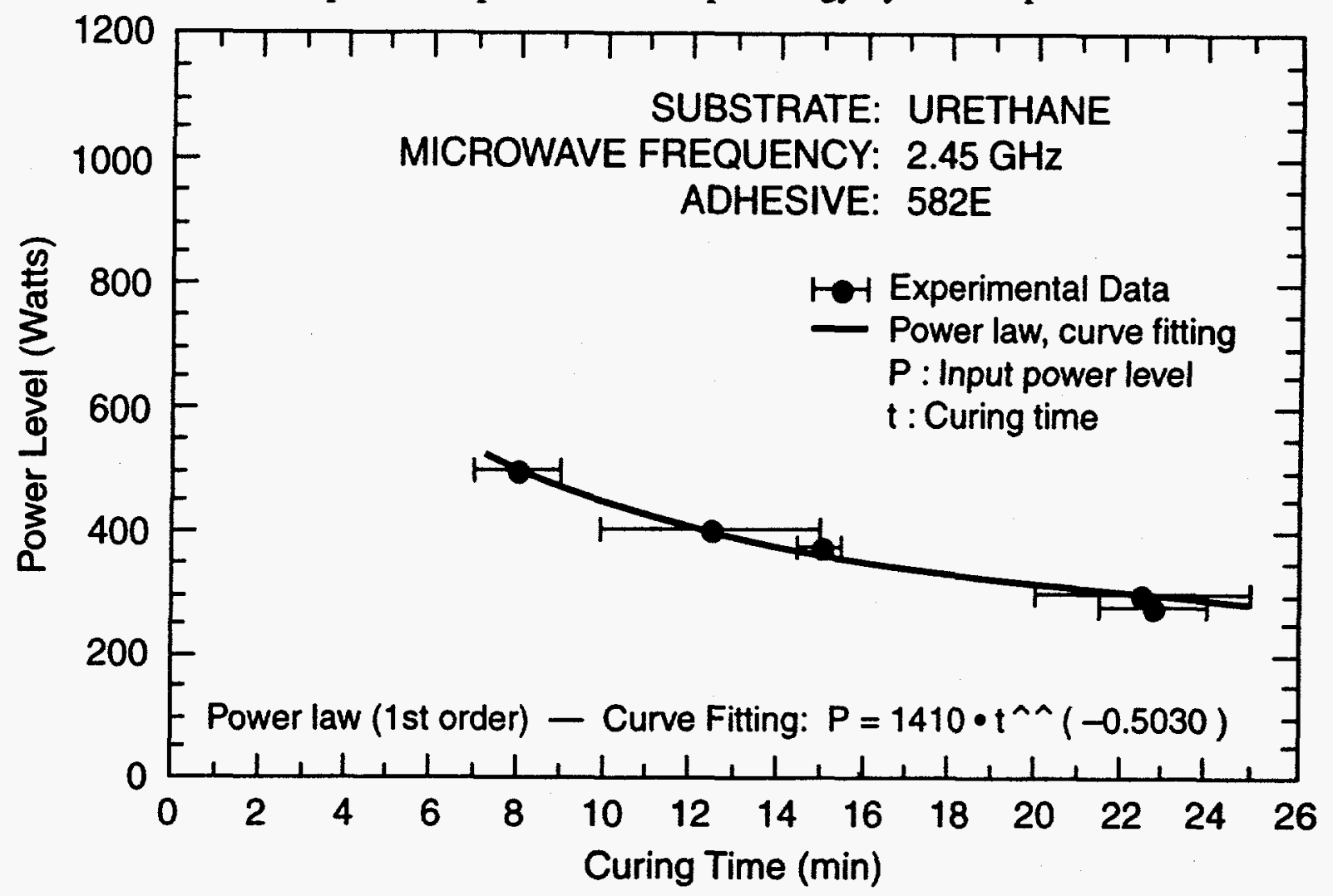

Figure 5. Cure Time vs. Power Level for the Adhesive on the Composite Substrates. Power Law Approximation.

\section{Mechanical Evaluation}

The characterization of the mechanical bond strength in the processed samples was determined using single lap-shear samples. In this evaluation, only urethane-glass substrates were studied. Table I gives the results of the single lap shear tests. Data is reported as maximum load carried and maximum total crosshead displacement (TCHD) rather than as stress and strain since for the single lap shear specimen configuration stress and strain cannot be directly correlated to machine displacements or load cell signals due to the complex test geometry, specimen geometry and off-set load path [8].

As seen in Table I, the ultimate tensile strength of the microwave processed samples cured for relatively short times was much lower than the conventionally processed samples. When the input power was reduced and the cure time lengthened to within the "moderate" range, the ultimate strengths of the microwave processed adhesives was nearly identical to that for the thermally cured adhesives. The microwave processed samples also had an increased TCHD and a decreased slope to the elastic loading curve. This indicates that the microwave processed samples may be less stiff than the conventionally cured samples and have a corresponding greater ductility. Mechanisms for this occurring are unknown, however it may be related to the difference in the thermal stressing of the substrate and adhesive by the two curing methods. 
Single Lap Shear Data for Single Frequency $(2.45 \mathrm{GHz})$ Microwave Processed Samples. Substrates are Urethane/Glass Composite. Adhesive is Goodrich 582E.

Ultimate Load is that required for failure. TCHD is the machine displacement at failure.

\begin{tabular}{|c|c|c|}
\hline & Ultimate Load (lbf) & TCHD (inches) \\
\hline $\begin{array}{c}\text { Conventional Cure } \\
45 \mathrm{~min}, 150^{\circ} \mathrm{C}\end{array}$ & 1420 & 0.065 \\
\hline \multicolumn{3}{|c|}{ MICROWAVE PROCESSED } \\
\hline $\begin{array}{l}\text { Short Cure } \\
12-14 \mathrm{~min}\end{array}$ & 959 & 0.085 \\
\hline $\begin{array}{l}\text { Moderate Cure } \\
22-30 \mathrm{~min}\end{array}$ & 1405 & 0.065 \\
\hline $\begin{array}{l}\text { Long Cure } \\
>30 \mathrm{~min}\end{array}$ & 1373 & 0.073 \\
\hline
\end{tabular}

It was noted that for fully cured joints, failure occurred at very high applied loads and by fiber tear of the composite substrate and seldom at the interface or within the adhesive. Bubble formation within the joints of the samples cured by microwave radiation for short times was also very prevalent indicating that the energy deposition rate was too high. This resulted in much weaker joints.

\section{VARIABLE FREQUENCY MICROWAVE RESULTS}

\section{Microwave Processing}

The first system evaluated was comprised of glass slides adhesively bonded using variable frequency microwave (Center frequency: $5.03 \mathrm{GHz}$ ) radiation over a range of input powers and frequency sweep ranges. An analysis of the data reveals that the epoxy cure time approximately followed the power law theory. In the high power region the experimental data indicates that the power varies as the negative power of the cure time, while for the lower input power region, the input power is inversely proportional to the exposure or cure time [6]. Through analyzing the experimental data presented in Figure 6, an appropriate empirical function was determined which describes this data to a high degree of accuracy. The empirical relationship which describes the observed data is a power law function with an exponent essentially of -0.21 and a constant term of approximately 308 . With the variable frequency microwave, much less input power was required to achieve curing than with the single frequency microwave. This is due to an improved coupling efficiency obtained by frequency sweeping.

Cure times below fifteen minutes, which correspond to input powers in the region of 200 watts produced samples with a significant volume fraction of bubbles in the as-cured adhesive. It should be noted that 200 watts is the maximum input power available in this specific VFM 
system. It is likely that the density of energy deposition into these samples occurred too rapidly resulting in excessive heating of the adhesive prior to sufficient crosslinking to prevent the out flow of the adhesive or generation of exotherm bubbles. As a result, there exists an upper threshold energy for curing this adhesive with this methodology. As the input power was decreased the time required for complete crosslinking was increased. At sufficiently low power inputs crosslinking would not be promoted. For this material system the lower threshold is below 125 watts. Therefore for this adhesive/substrate material combination, VFM system and processing conditions the effective curing range of the adhesives is between 125 and 200 watts and the complete cure can be achieved in as little as fifteen minutes. It should be noted that the upper and lower power threshold for the variable frequency processing were much lower than for the single frequency processing. This indicates that from an energy standpoint, variable frequency processing may be much more efficient for this type of operation.

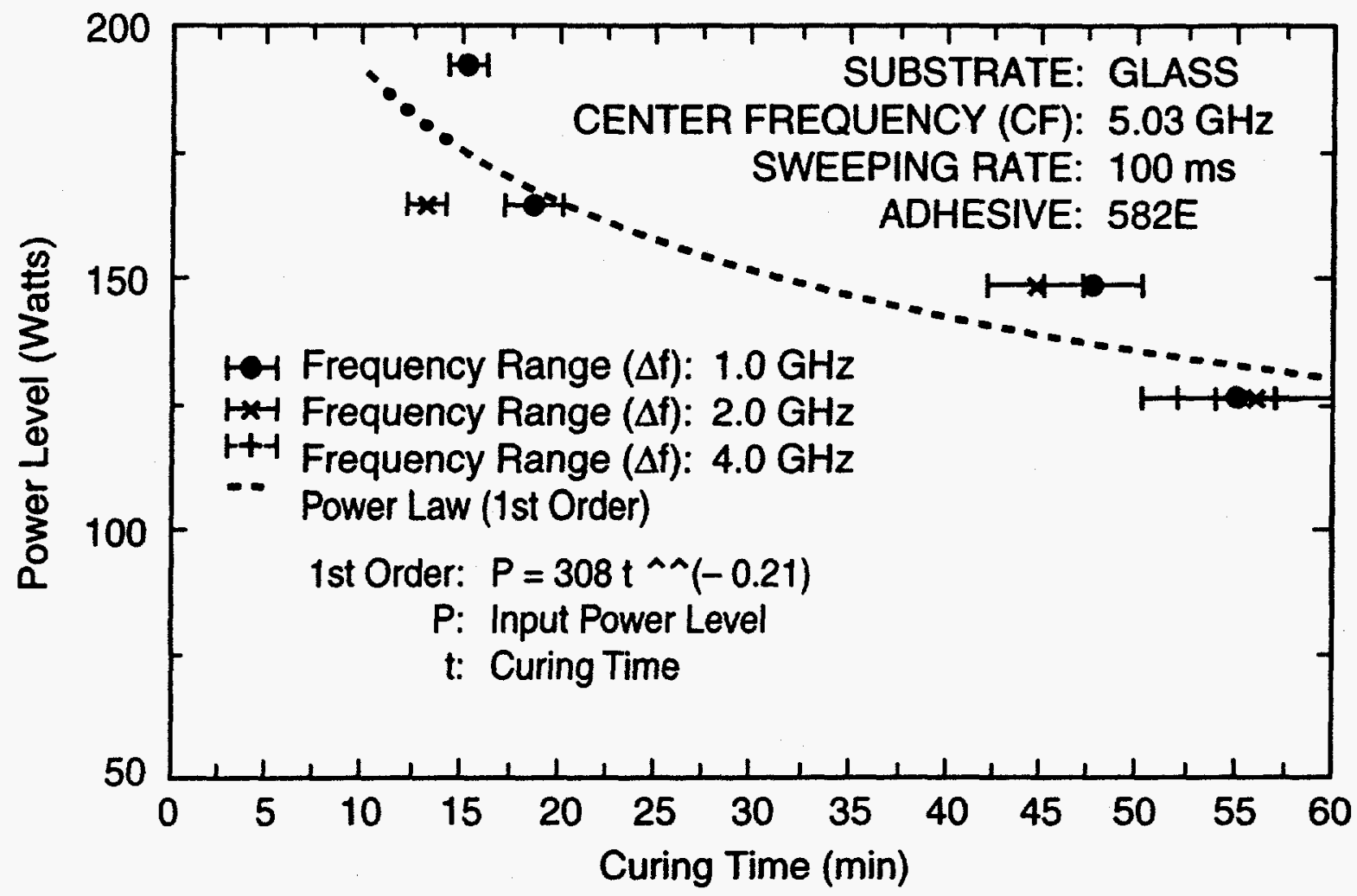

Figure 6. Cure Time vs. Power Level for the Glass Substrates. Power Law Approximation.

The second system evaluated was the glass fiber reinforced isocyanurate composite previously described. The composite was adhesively bonded using variable frequency microwave (Center Frequency: $5.03 \mathrm{GHz}$ ) radiation over the range of input powers and frequency sweep ranges previously indicated. An analysis of the data shows that this material system approximately follows the power law theory which is shown in Figure 7. The empirical function which best describes this data is a power law function with an exponent of -0.49 and a constant term of 604 . This function easily fit the data from all three frequency ranges. With this exponent (approximately -0.5 ) a doubling of the input power will result in a four fold reduction in the curing time. A tripling of the input power will result in a nine fold reduction in the curing time. Any increase in input power will result in a much more drastic reduction in the required curing time for this adhesive/substrate material system. The lowest cure time 
possible, however, is always limited by the maximum input energy threshold which is a function of the specific material systems being studied and the frequency being utilized.

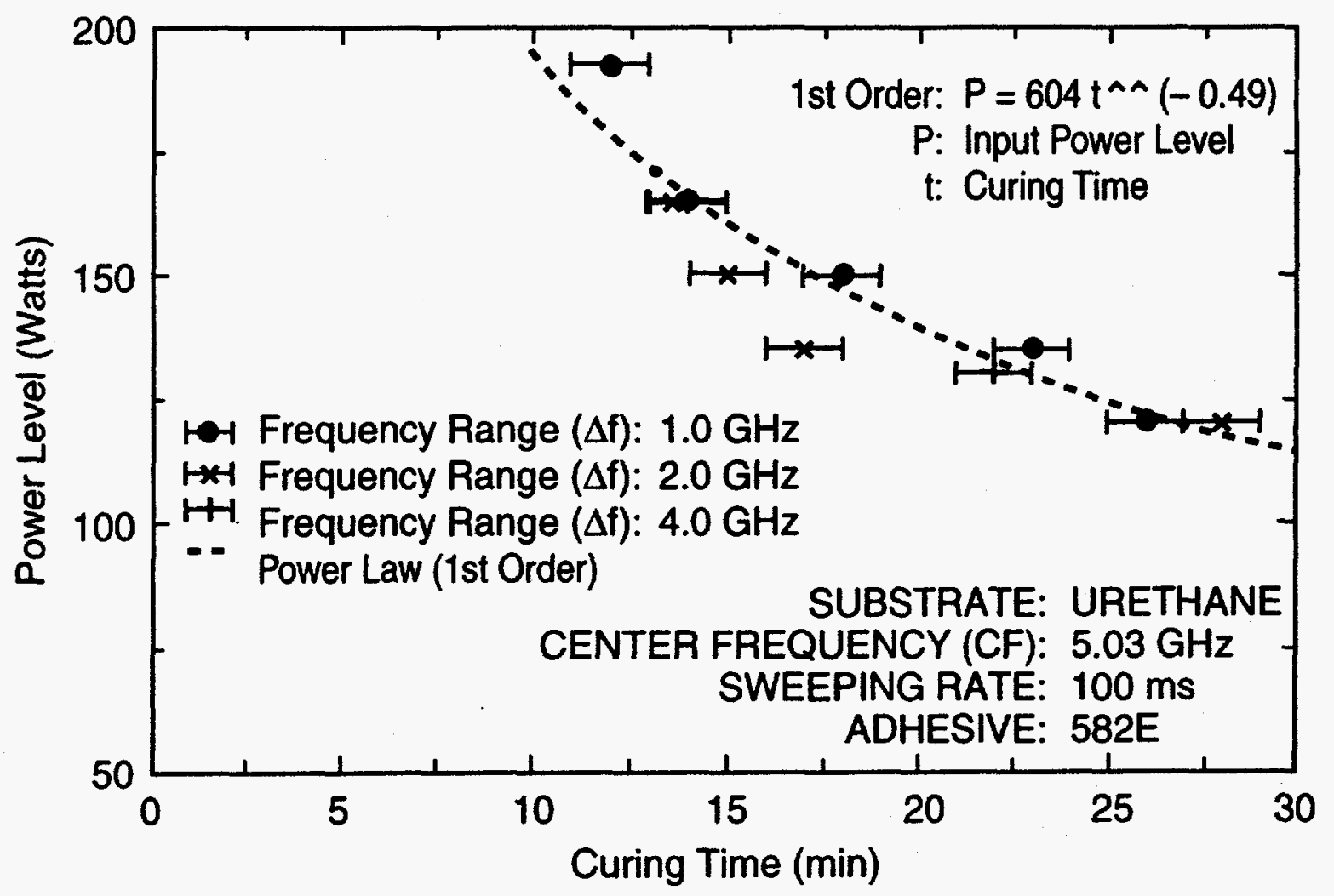

Figure 7. Cure Time vs. Power Level for the Composite Substrates. Power Law Approximation.

Input power of about 200 watts, which corresponds a to cure time below twenty-two minutes, is the maximum power limit due to the formation of bubbles in the adhesive. The lower limit for complete crosslinking for this material system is below approximately 150 watts. Therefore for this material and microwave system the effective curing range of the adhesives for these conditions is between 150 and 200 watts and the complete cure can be achieved in as little as fifteen minutes. This is significantly longer than the eight to twelve minutes required by the single frequency microwave but may be related to the lower power level available in the VFM used in this work. The time difference between the two methods may also be attributable to frequency being applied. ( $2.45 \mathrm{GHz}$ for single frequency and a center frequency of 5.03 $\mathrm{GHz}$ for variable frequency)

No acceptable comparison can be established between the $5.03 \mathrm{GHz}$ center frequency in the VFM processing and the $2.45 \mathrm{GHz}$ frequency used in the fixed frequency processing. Even if the VFM center frequency were the same as the fixed frequency, a direct quantitative correlation could not be established between the two processing methods due to differences in the physics of the two processes. For fixed frequency microwave processing, the sample has to be located at the same place inside the microwave cavity. The determination of the optimum specimen placement in the oven has to be conducted by trial and error preliminary screening trials. Once a "good" location in the oven is determined, each batch of processed samples must be properly placed in that selected location. In this way, samples processed by fixed frequency microwaves can be compared to each other. For variable frequency microwave processing, the 
location of the samples inside of the cavity is not important which makes this technology more robust for manufacturing environments. Therefore, a direct correlation between these two microwave processing methods is not possible due to the location sensitivity of the single frequency processed samples. We will, however, draw a loose comparison between these two methods. This is done because neither of these technologies will be transferable to the manufacturing world unless comparisons are made which have understandable significance.

\section{Mechanical Evaluation}

The characterization of the bond strength in the processed samples was determined using the same test configuration and equipment as was used in evaluating the single frequency microwave cured samples. In this evaluation, only composite substrates were studied. Table II gives the results of the single lap shear tests. Data is reported as ultimate load (UL) carried and maximum total crosshead displacement (TCHD) rather than as stress and strain for reasons previous explained [8]. A detailed description of the mechanical evaluation results from fixed frequency $(2.45 \mathrm{GHz})$ processing can be found in previous work $[9,10]$.

\section{Table II}

Single Lap Shear Data for Single and Variable Frequency Microwave Processed Samples Substrates are Urethane/Glass Composite. Adhesive is Goodrich 582E.

Ultimate Load is that required for failure and is expressed in pounds.

TCHD is the machine displacement at failure expressed in inches.

\begin{tabular}{|c|c|c|c|c|c|c|}
\hline & \multirow{2}{*}{\multicolumn{2}{|c|}{$\begin{array}{l}\text { Single Frequency } \\
\mathrm{SF}=2.45 \mathrm{GHz}\end{array}$}} & \multicolumn{4}{|c|}{ Variable Frequency $\mathrm{CF}=5.03 \mathrm{GHz}$} \\
\hline & & & \multicolumn{2}{|c|}{$\mathrm{FR}=1.00 \mathrm{GHz}$} & \multicolumn{2}{|c|}{$\mathrm{FR}=2.00 \mathrm{GHz}$} \\
\hline & UL & TCHD & UL & TCHD & UL & TCHD \\
\hline Short Cure & & & & & & \\
\hline $12-14 \mathrm{~min}$ & 950 & 0.085 & 1005 & 0.040 & 960 & 0.041 \\
\hline Moderate $\mathrm{Cl}$ & & & & & & \\
\hline $22-30 \mathrm{~min}$ & 1405 & 0.065 & 1390 & 0.065 & 1400 & 0.072 \\
\hline $\begin{array}{l}\text { Long Cure } \\
>30 \mathrm{~min}\end{array}$ & 1373 & 0.073 & & & & \\
\hline
\end{tabular}

Conventional Cure: $\mathrm{UL}=1420 \mathrm{lbf}, \mathrm{TCHD}=0.065$ inches

As seen in Table II and Figure 8, the samples processed by variable frequency microwave irradiation for 12 to 14 minutes had strengths and total elongations far below those of conventionally processed samples or the microwave processed samples cured for longer periods of time at lower energies. Post failure analysis revealed that these samples contained bubbles characteristic of a material that underwent too high of an energy deposition during curing. This same phenomenon has been previously noted [7] where the same adhesive was thermally cured at too high of a temperature. In that work, the exotherm of the epoxy produced the bubbles. 


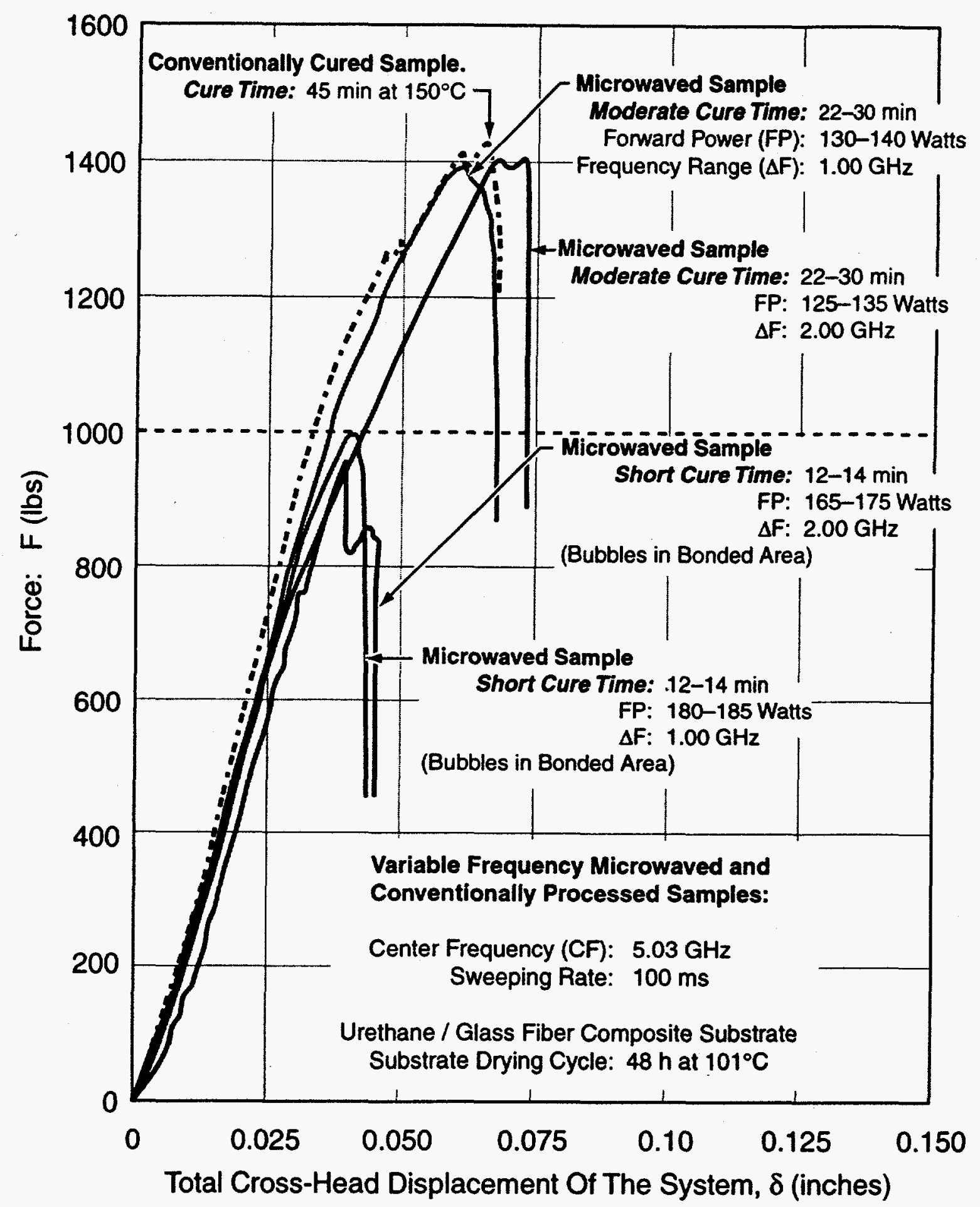

Figure 8. Typical Single Lap Shear Curves for Variable Frequency Microwave Processed Samples. Comparison to Thermally Cured Specimens is made.

The samples processed by lower input energies for longer periods of time had ultimate strengths almost exactly identical to those of the thermally cured samples. The total elongation (i.e. ductility, lack of stiffness) of the samples microwaved between 22 and 30 minutes was slightly greater than that of thermally cured samples. Thus equivalent failure strengths and increased ductility was noted at cure times that were between $1 / 2$ and $2 / 3$ those required for 
conventional curing. The increased ductility for variable frequency processed samples was not as extensive as that noted for single frequency processed samples. Further studies are required in this area to find an explanation for this observation.

\section{CONCLUSIONS}

1. The resultant energy deposition profile is more uniform for variable frequency microwave systems than for fixed frequency microwave systems. As a result, VFM curing is independent of sample placement in the microwave oven while fixed frequency microwave curing is highly sample position sensitive. Contrary to fixed frequency systems, samples cured in VFM ovens are processed with less generation of localized hot or burn spots which result in better quality bonds.

2. Variable frequency microwave technology represents a valuable alternative method for rapidly curing thermoset adhesives at low input power levels. For the substrates and adhesive used in this project, surprisingly low levels of forward (input) power were required for processing of the samples.

3. The application of microwave technology for joining of substrates using epoxy based adhesives significantly reduces the curing time to only a third to a quarter of the conventional cure time. This is accomplished while maintaining equivalent values of the ultimate tensile strength measured through the single lap shear test. This was observed for both fixed and variable frequency microwave technologies.

4. Microwave processed samples (fixed and variable frequency), when tested as single lap shear specimens exhibit less rigidity and more plasticity than conventionally processed samples.

5. Coupling of the Goodrich EXP 582E epoxy based adhesive to the fixed and variable frequency microwave radiation is extremely efficient for all the frequencies applied in this study.

6. Variable frequency microwave processing appears to yield a slight reduction in the required adhesive cure time when compared to processing by the application of a fixed frequency microwave source.

7. This technology may be extended to multiple-layered panels or components.

\section{ACKNOWLEDGEMENTS}

Research was performed at Oak Ridge National Laboratory and sponsored by the Office of Transportation Materials, U.S. Department of Energy, under contract No. DE-AC05-96OR22464 with Lockheed Martin Energy Research Corporation. 


\section{REFERENCES}

1. Office of Transportation Materials, Materials for Lightweight Vehicles Program Plan, United States Department of Energy, (1992).

2. A.D. Surrett, R.J. Lauf, F.L. Paulauskas and A.C. Johnson, MRS Symposium Proceedings, 347, 691 (1994).

3. A.C. Johnson, R.J. Lauf and A.D. Surrett, MRS Symposium Proceedings, 347, 453 (1994).

4. R.J. Lauf, F.L. Paulauskas and A.C. Johnson, 28th Microwave Symposium Proceedings International Microwave Power Institute, 150 (1993).

5. R.J. Espinosa, A.C. Johnson, L.T. Thigpen, W.A. Lewis, C.A. Everleigh and R.S. Garard, 28th Microwave Symposium Proceedings - International Microwave Power Institute, 26 (1993).

6. F.L. Paulauskas, T.T. Meek and C.D. Warren, SAMPE International Technical Conference Proceedings, 27, 114 (1995).

7. C.D. Warren, R.G. Boeman and F.L. Paulauskas, 1994 DOE Contractors Coordination Meeting Conference Proceedings, 2, 1 (1994).

8. R.G. Boeman and C.D. Warren, 10th Annual ASM/ESD Advanced Composites Conference Proceedings, 473 (1995).

9. F.L. Paulauskas, T.T. Meek and C.D. Warren, MRS Symposium Proceedings, 430 (1996) In press in this proceeding.

10. F.L. Paulauskas and T.T. Meek, MRS Symposium Proceedings, 347, 743 (1994).

\section{DISCLAIMER}

This report was prepared as an account of work sponsored by an agency of the United States Government. Neither the United States Government nor any agency thereof, nor any of their employees, makes any warranty, express or implied, or assumes any legal liability or responsibility for the accuracy, completeness, or usefulness of any information, apparatus, product, or process disclosed, or represents that its use would not infringe privately owned rights. Referprocess disclosed, or represents that its use would nocess, or service by trade name, trademark, manufacturer, or otherwise does not necessarily constitute or imply its endorsement, recommanufact or favoring by the United States Government or any agency thereof. The views and opinions of authors expressed herein do not necessarily state or reflect those of the United States Government or any agency thereof. 